\title{
Mortality rate of patients with cystic fibrosis on the waiting list and within one year after lung transplantation: a survey of Italian CF centers
}

Beatrice Borchi', Marisol Barao Ocampo ${ }^{2}$, Giuseppe Cimino ${ }^{3}$, Giovanna Pizzamiglio ${ }^{4}$, Silvia Bresci ${ }^{1}$, Cesare Braggion ${ }^{5^{*}}$ and the Italian Cystic Fibrosis Lung Transplantation Group

\begin{abstract}
Background: Cystic Fibrosis (CF) Centers are involved in the decisions regarding the eligibility of CF patients with end-stage lung disease and timing for inclusion on waiting lists (WL) for lung transplantation (LT). There are currently no data on the mortality rates of Italian CF patients on WL and during the first year after LT and we aimed to assess these outcomes by surveying the CF Centers.

Methods: A survey was sent to Italian CF Centers which were requested to report the age at which all CF subjects included on the WL between 2010 and 2014 were included on the list, admitted to either standard or urgent LT, or had died either while on the WL or within the first 3 and 12 months after LT. All outcomes were recorded by December 31, 2015.

Results: Two hundred fifty-nine CF subjects were included on the WL during the 5-year study period. The mortality rate during the WL was $19.3 \%$ and was not associated with sex, age at inclusion on the WL or standard or urgent access to LT. 159 (61.4\%) subjects underwent LT, 46 (28.9\%) with urgent procedure. Deaths within the first 3 and 12 months after LT were significantly more prevalent in individuals who underwent urgent LT compared to those with standard LT $(p<0.01)$.

Conclusions: The mortality of Italian CF patients, included in our survey, was about twice that reported by the National Transplant Center for all LT indications, including CF, during the same time period and despite the introduction of urgent LT. The latter was associated with an unfavorable early outcome compared to standard LT.
\end{abstract}

\section{Background}

Lung transplantation (LT) is offered to subjects with cystic fibrosis (CF) with end-stage lung disease as a potential life-extending and life-improving treatment $[1,2]$. Different predictive models for mortality have been proposed but the optimal timing for listing of CF individuals for LT is a complex task for the CF specialist [3-5].

In addition to measures which attempt to deal with the shortage of suitable lung donors, different organ allocation policies, based on medical urgency, have been

\footnotetext{
* Correspondence: cesare.braggion@meyer.it

${ }^{5}$ Cystic Fibrosis Center, Meyer Children's Hospital, Viale Pieraccini 24, 50139

Florence, Italy

Full list of author information is available at the end of the article
}

proposed to avoid long waiting times and death either while on the waiting list (WL) or during the first year after LT. The lung allocation score (LAS) was implemented to prioritize patients in the United States [6]. Dedicated emergency LT procedures have been proposed as strategies in France and Italy for patients in extremely urgent medical conditions, such as those requiring mechanical ventilation (MV) or extracorporeal membrane oxygenation (ECMO) [7-10]. The urgent LT procedure is proposed only for patients already wait-listed in Italy $[9,10]$. In France the "high emergency waiting list" is proposed for patients with an abrupt worsening of their respiratory function, requiring invasive mechanical ventilation (MV) and/or ECMO or who are at risk of imminent invasive

(c) The Author(s). 2018 Open Access This article is distributed under the terms of the Creative Commons Attribution 4.0 International License (http://creativecommons.org/licenses/by/4.0/), which permits unrestricted use, distribution, and 
MV or of having severe pulmonary hypertension [7, 8]. The inclusion on the regular waiting list was not an inclusion criterion in France, as recorded for $51.5 \%$ of patients in a recent nation-based study [8].

Italian CF Centers are involved in LT programs, by determining the timing for inclusion on the WL and by caring for patients while they await surgery. Listing criteria were the same as those suggested in the international literature $[1,2]$.

Due to the lack of data on the mortality rate of Italian CF subjects on the WL and during the first year after LT, we surveyed these outcomes, as recorded by Italian CF Centers.

\section{Methods}

This retrospective cross-sectional survey included 22 Italian CF Centers. All CF subjects were referred to $\mathrm{CF}$ Centers to assess the timing for their inclusion on the WL and optimize their medical treatment during their wait. They were then referred to the Lung Transplant Centers, which established their eligibility for LT and inclusion on the WL. According to the Italian Cystic Fibrosis Register (ICFR) Report 2010, our survey addressed 4159 children and adult CF patients in 2010 [11].

CF Centers were asked to record all WL subjects between 2010 and 2014. The survey covered the following demographic and outcome data: year of inclusion on the WL, sex, age at inclusion on the WL, age at time of standard or urgent LT, age at time of death occurring either while on the WL or within 3 and 12 months after transplant. The outcomes were considered as of December 31, 2015.

CF Centers were requested to review systematically the medical records of all subjects included on the WL to avoid missing data. According to our aims, we chose to collect data only on mortality before and during the first year after LT.

Eleven Lung Transplant Centers were active in Italy in the period 2010-2014 [12]. In October 2010, under the supervision of the National Transplant Center, all Lung Transplant Centers created and agreed on a common protocol to identify patients requiring lung transplant priority $[9,10]$. The Italian Urgent Lung Transplant program (IULTp) is reserved for patients younger than 50 years, who moved from the standard lung transplant program to urgent LT, if they required mechanical ventilation and/or extracorporeal lung support. Exclusion criteria are sepsis, multiorgan failure, hemorrhagic shock, neurological damage and invasive respiratory support lasting more than 14 days. Since 2010 both the standard LT program and the IULTp have been active in Italy. Priority of the IULTp can last 3 weeks. All available lungs in the country must be considered for urgent transplant first.
The yearly number of LTs, deceased patients on waiting lists and patients still on the WL for all LT indications, including patients with $\mathrm{CF}$, are recorded in the Reports of the National Transplant Center [12]. Data on single LT indications and LT modality (single/bilateral/ heart-lung) are not available.

We considered also the number of LTs and age at the surgical procedure recorded by the ICFR in Italy during 2010-2014, to compare these data with those of the CF subjects included in our study $[11,13]$. The ICFR did not collect clinical data of CF individuals at time of inclusion on the WL, during the waiting time, at the time of transplantation, during the perioperative period or in the follow-up after the LT.

The study protocol was approved by the Ethics Committee of the Hospitals involved in the survey.

Descriptive statistics and comparisons between groups for continuous variables were performed according to normal distribution tests. Kruskal-Wallis analysis of variance was used to compare variables among three groups of individuals according to their age. The Mann-Whitney test was used to compare demographic data and outcomes of subjects listed in the standard versus the urgent LT programs.

Differences in proportions of categorical data were assessed using the Chi-square test. Statistical significance was defined as $p<0.05$.

\section{Results}

16/22 (72.7\%) CF Centers sent data on CF individuals included on the WL during the 5-year study period. The survey covered $82.4 \%$ of subjects included in the 2010 ICFR Report [11].

Two hundred fifty-nine subjects were included on the WL: the median (interquartile range) age was 30.4 (22.1-37.5) years (Table 1). Three subjects were removed from the WL because of improved clinical condition after 2.1, 0.8 and 0.8 years, respectively. Two subjects, retransplanted during the study period, were considered only for their first LT. We recorded 50 deaths (19.3\%) during patients' time on the WL, 159 lung transplants $(61.4 \%)$ and $47(18.1 \%)$ subjects still on the WL at December 31, 2015 (Table 1).

CF individuals were grouped by age into three groups at the time of inclusion on the WL, with the aim of comparing outcomes between pediatric patients, young adults and subjects older than 30 years. Table 1 shows that more females were recorded among pediatric subjects compared to adults either younger or older than 30 years, but the difference was not statistically significant $(p=0.061)$. There were no statistically significant differences in number of deaths, LTs, subjects still on the WL, and duration of WL between deceased and transplanted subjects in the three groups. The waiting 
Table 1 Characteristics and outcomes of 259 subjects with cystic fibrosis included on the waiting list for lung transplantation during 2010-2014

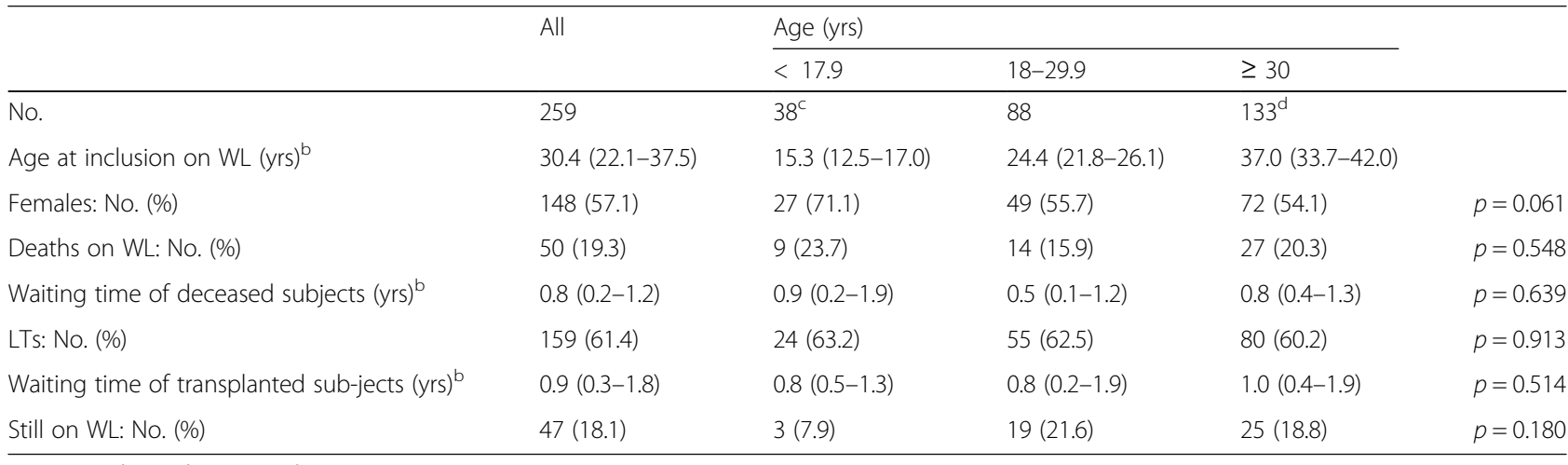

WL waiting list, $L T$ lung transplantation

a outcomes were considered until December 31, 2015

${ }^{\mathrm{b}}$ data are reported as median and interquartile range

two subjects were removed from the WL after 2.1 and 0.8 years

${ }^{d}$ one subject was removed from the WL after 0.8 years

time of deceased subjects on the WL was not different from that of transplanted subjects (Table 1).

Table 2 compares demographic data and outcomes of 57 individuals listed in the IULTp to 202 patients listed only in the standard LT program. There were no differences in sex, age at inclusion on the WL and mortality rate during WL between the two groups. The percentage of subjects who waited less than 3 months for surgery was significantly different in the two groups $(43.9 \%$ vs $3.5 \%: p<0.001)$. Deaths within 3 and 12 months were more frequent in subjects who required an urgent procedure compared to those undergoing standard LT (19.3 and $24.6 \%$ vs 6.4 and $8.9 \%, p<0.01$ and $p<0.01$, respectively).

The National Transplant Center reported 107 LTs in 2010, 120 in 2011, 114 in 2012, 141 in 2013 and 126 in 2014, including single, bilateral and heart-lung LTs. The mean mortality of those patients on the WL was $11.5 \%$ in $2010,10.2 \%$ in $2011,12.1 \%$ in $2012,10.9 \%$ in 2013 and 9.3\% in 2014 for all indications for LT [12].

The ICFR reported that there were 20 LTs in CF patients in 2010, 36 in 2011, 25 in 2012, 40 in 2013 and 34 in 2014 [11, 13]. According to the Reports of the National Transplant Center, the primary disease of 155/608 transplant recipients was cystic fibrosis (25.5\%) [11-13]. Data on mortality of patients on the WL, number of either urgent or standard LTs, data on mortality after LT were not reported by the ICFR [11-13].

\section{Discussion}

The major findings of our study are the $19.3 \%$ mortality rate of those CF patients on the WL and the less favorable outcome soon after LT of those patients who

Table 2 Comparison of demography and outcomes between subjects listed for urgent lung transplantation program and those listed for standard lung transplantation program ${ }^{a}$

\begin{tabular}{|c|c|c|c|}
\hline & Urgent lung transplantation program & Standard lung transplantation program & \\
\hline No. & 57 & $202^{c}$ & \\
\hline Females: No. (\%) & $34(59.6)$ & $114(56.4)$ & $p=0.665$ \\
\hline Age at inclusion on WL (yrs) ${ }^{b}$ & $27.6(23.4-36.7)$ & $30.5(21.8-37.6)$ & $p=0.826$ \\
\hline Deaths on WL: No. (\%) & $11(19.3)$ & $39(19.3)$ & $p=0.998$ \\
\hline Waiting time of deceased subjects $(y r s)^{b}$ & $0.4(0.2-0.9)$ & $0.8(0.3-1.4)$ & $p=0.127$ \\
\hline LTs: No. (\%) & $46(80.7)$ & $113(55.9)$ & $p<0.001$ \\
\hline Waiting time of transplanted subjects $(y r s)^{b}$ & $0.2(0.1-1.0)$ & $1.0(0.6-1.9)$ & $p<0.001$ \\
\hline Subjects waiting less than 3 months before LT: No. (\%) & $25(43.9)$ & $7(3.5)$ & $p<0.001$ \\
\hline $\begin{array}{l}\text { Transplanted subjects deceased during the first } 3 \text { months } \\
\text { after LT: No. (\%) }\end{array}$ & $11(19.3)$ & $13(6.4)$ & $p<0.01$ \\
\hline $\begin{array}{l}\text { Transplanted subjects deceased during the first } 12 \text { months } \\
\text { after LT: No. (\%) }\end{array}$ & $14(24.6)$ & $18(8.9)$ & $p<0.01$ \\
\hline
\end{tabular}

$W L$ waiting list, $L T$ lung transplantation

a outcomes were considered until December 31, 2015

bdata are reported as median and interquartile range

$c^{c}$ three subjects were removed from the WL after 2.1, 0.8 and 0.8 years 
underwent urgent LT, when compared with those undergoing standard procedure.

The introduction of LAS in the USA has helped to reduce the mortality rate for CF patients on the WL from 22 to 29 to $12.9 \%$ and benefited survival after LT in recipients with CF [5, 6, 14-16]. Preliminary reports have recorded a decrease in mortality of CF patients on WL from 22.1 to $13.7 \%$, comparing 1-year before and after introduction of LAS in Germany in 2011 [17].

Both dedicated emergency programs in France and Italy were associated with a decrease in mortality of patients on the WL for all indications: in France from 8.6\% in 2006 to $3.5 \%$ in 2011, in Italy from a range of 11.5$14.7 \%$ during $2005-2009$ to a range of $9.3-12.1 \%$ during 2010-2014 [8-10, 12]. Unfortunately, data about outcomes of different underlying lung diseases is not available in Italy. The mortality rate of CF subjects on the WL in our study remained high, being nearly double that recorded for all indications for LT, including CF, in the same time period, during which the urgent procedure was implemented [15]. The findings on mortality rate in CF may suggest that a different organ allocation policy should be considered in Italy to improve survival for subjects with cystic fibrosis who are on the WL and for those who receive lung transplantation.

A late inclusion in WL and therefore a poor selection of CF patients should be unlikely for the standard LT, since we found that the waiting time was similar in deceased and transplanted individuals. Inclusion and exclusion criteria on the WL were not different from those reported in the literature $[1,2]$. However, we found that the waiting time of subjects undergoing urgent LT was significantly less compared to those undergoing standard LT. A waiting time of less than 3 months was recorded in $43.9 \%$ of subjects who were moved from standard to urgent LT. An unpredictable, rapid decline in lung function can occur in some patients with $\mathrm{CF}$ and moving from standard to urgent LT represents the only opportunity for a lung transplant in these patients.

Donor shortage and the inadequate number of LTs are also reasons for mortality rates in CF patients waiting for a transplant. The ratio between number of LTs and number of subjects on the active WL was 312/490 (0.64) in France, 327/581 (0.56) in Germany and 120/561 $(0.21)$ in Italy in 2011: these figures were associated with different WL durations [8-10, 12, 17, 18].

For patients who moved from standard to urgent LT, IULTp resulted in LT in $80.7 \%$ of the cases and should be considered an efficient strategy, considering that mortality while on the WL (19.3\%) was the same as that for patients in the standard program (19.3\%). The IULTp therefore offered an opportunity for LT to seriously ill CF patients who would have otherwise died while on the WL. However, the IULTp was associated with increased mortality within the first year after LT. The urgent LT program in France showed similar results: the overall in-hospital mortality rate was $29.4 \%$ for 95 transplanted subjects, with extracorporeal membrane oxygenation (ECMO) prior to LT being the sole independent mortality risk factor [8]. The high mortality associated with IULTp should be interpreted cautiously in the context of the most critical conditions in which it was used. A high LAS score was also associated with an increased risk of death after LT in cystic fibrosis patients [19]. Prospective research is needed to assess whether timing, duration and modality of ECMO and associated invasive respiratory support could be modified to decrease the mortality rate after LT.

The main limitation of our survey is that we included only data on the mortality rate of CF patients on the WL and during the first year after LT. We did not record the clinical characteristics of CF individuals at the time of inclusion on the WL, the deterioration of lung disease during the waiting time, lung donor characteristics, the modality and duration of invasive support for patients in the urgent program before and after LT, all of which are risk factors affecting survival before and after LT $[1-5,8,14,15]$. Many of these clinical data were recorded by the Transplant Centers, but were not included in our survey. Neither the ICFR nor the National Transplant Center collected or reported this important clinical information. The ICFR recently reported that $10 \%$ of transplanted subjects had missing data on outcomes after LT [13]. Probably some patients were followed up only by the Transplant Centers once they received a LT. A prospective study should be designed which involves both CF Centers and Lung Transplant Centers to collect clinical data for assessing which risk factors affect survival before and after LT.

Our survey covered $82.4 \%$ of CF subjects in Italy during our study period and our mortality rates could be underestimated. The ICFR recently reported that $155 \mathrm{CF}$ patients received a LT in the same 5 -year period $[11,13]$. Our study reported 159 LTs for 259 subjects included on the WL. Although a bias of missing data cannot be excluded, our study recorded mortality data of all 259 subjects included on the WL between 2010 and 2014.

\section{Conclusions}

In conclusion, despite some limitations, this study is the first attempt to evaluate mortality of Italian CF patients while they are on the waiting list for lung transplantation and within 3 and 12 months after they have received either urgent or standard LTs. In spite of the introduction of emergency LT in Italy, the mortality rate of CF patients on the WL was twice that for all LT indications, including CF, and higher than that recorded in other countries for the same disease. The urgent procedure was associated with a less favorable outcome soon after LT. 
To decrease the mortality of CF patients on the WL and to improve outcomes after LT, health authorities need to acknowledge different aspects of the LT program, from the shortage of suitable lung donors to organ allocation policies. The use of marginal grafts, the reconditioning of grafts with poor function and from donation after cardiac death and living lobar transplant have been developed to increase the number of LTs. The current organ allocation policy in Italy should also be critically evaluated in relation to the results obtained in CF subjects: disease-specific parameters should be considered for waitlist death risk and transplant benefit to give priority to LT. A national registry that is linked with the transplant centers and which collects demographic and clinical data of all subjects at the time of their inclusion on the waiting list, as well as outcome after LT, is a top priority for improving the organ allocation policy for all LT indications in Italy.

(Manuscript: "Mortality rate of patients with cystic fibrosis on the waiting list and within one year after lung transplantation: a survey of Italian CF Centers")

\section{Acknowledgements}

The Italian Cystic Fibrosis Lung Transplantation Group includes the directors of the CF Centers and their coworkers, which collected the data required by the survey.

The Italian Cystic Fibrosis Lung Transplantation Group includes:

- Barbara Messore, MD, Cystic Fibrosis Center - Adult Unit, Interstitial and Rare Lung Diseases Unit, Ospedale San Luigi Gonzaga, Orbassano, Turin;

- Carlo Albera, MD, Cystic Fibrosis Center - Adult Unit, University of Turin, Department of Clinical and Biological Sciences, Interstitial and Rare Lung Diseases Unit, Ospedale San Luigi Gonzaga, Orbassano, Turin;

- Elisabetta Bignamini, MD, Cystic Fibrosis Center, Regina Margherita Children's Hospital, Turin;

- Luca Zito, MD, Cystic Fibrosis Center, Regina Margherita Children's Hospital, Turin;

- Maria Pappalettera, MD, Cystic Fibrosis Center - Adult Unit, Fondazione IRCCS Ca' Granda, Ospedale Maggiore Policlinico, Milan;

- Carla Colombo, MD, Cystic Fibrosis Center - Paediatric Unit, Fondazione IRCCS Ca' Granda, Ospedale Maggiore Policlinico, Milan;

- Valeria Daccò, MD, Cystic Fibrosis Center - Paediatric Unit, Fondazione IRCCS $\mathrm{Ca}^{\prime}$ Granda, Ospedale Maggiore Policlinico, Milan;

- Laura Minicucci, MD, Cystic Fibrosis Center, G. Gaslini Institute, Genoa;

- Federico Cresta, MD, Cystic Fibrosis Center, G. Gaslini Institute, Genoa;

- Sonia Volpi, MD, Cystic Fibrosis Center, Azienda Ospedaliera Universitaria, Verona;

- Fiorella Battistini, MD, Cystic Fibrosis Center, Ospedale M. Bufalini, Cesena;

- Benedetta Mannozzi, MD, Cystic Fibrosis Center, Ospedale M. Bufalini, Cesena;

- Giovanna Pisi, MD, Cystic Fibrosis Center, Azienda Ospedaliero Universitaria, Parma;

- Francesco Longo, MD, Cystic Fibrosis Center, Azienda Ospedaliero Universitaria, Parma;

- Annalisa Cavallo, MD, Infectious Disease Unit, Azienda OspedalieroUniversitaria di Careggi, Florence;

- Anna Silvia Neri, MD, Cystic Fibrosis Center, Meyer Children's Hospital, Florence;
- Manuel Murciano, MD, Cystic Fibrosis Center - Adult Unit, Department of Medicine and Infectious Diseases, University Umberto I, Rome;

- Vincenzina Lucidi, MD, Cystic Fibrosis Center, Bambino Gesù Children's Hospital, Rome;

- Enza Montemitro, MD, Cystic Fibrosis Center, Bambino Gesù Children's Hospital, Rome;

- Valeria Raia, MD, Cystic Fibrosis Center - Paediatric Unit, Azienda Ospedaliera Universitaria, University Federico II, Naples;

- Antonella Tosco, MD, Cystic Fibrosis Center - Paediatric Unit, Azienda Ospedaliera Universitaria, University Federico II, Naples:

- Vincenzo Carnovale, MD, Cystic Fibrosis Center - Adult Unit, Geriatria, University Federico II, Naples;

- Paola Iacotucci, MD, Cystic Fibrosis Center - Adult Unit, Geriatria, University Federico II, Naples;

- Antonio Manca, MD, Cystic Fibrosis Center, AOU Policlinico, Bari;

- Giuseppina Leonetti, MD, Cystic Fibrosis Center, AOU Policlinico, Bari;

- Giuseppe Magazzù, MD, Cystic Fibrosis Center, Azienda Ospedaliera Universitaria Policlinico G. Martino, Messina:

- Maria Cristina Lucanto, MD, Cystic Fibrosis Center, Azienda Ospedaliera Universitaria Policlinico G. Martino, Messina;

- Mirella Collura, MD, Cystic Fibrosis Center, ISMEP-ARNAS Civico, Palermo;

- Francesca Ficili, MD, Cystic Fibrosis Center, ISMEP-ARNAS Civico, Palermo.

\section{Funding}

This research did not receive any specific grant from funding agencies in the public, commercial or not-for-profit sectors.

\section{Availability of data and materials}

All data generated or analysed during this study are included in this published article.

\section{Authors' contributions}

All the authors contributed to the research design, writing of the paper, collection of data at their Center and data analysis. All authors read and approved the final manuscript. The Italian Cystic Fibrosis Lung

Transplantation Group includes the directors of the CF Centers and their coworkers, which collected the data required by the survey. They read and approved the contents of the final manuscript.

\section{Ethics approval and consent to participate}

The study protocol was approved by the Ethics Committee of the Hospitals involved in the survey. Informed consent to participate in the study was obtained from participants or their parent or legal guardian in the case of children under 16 .

\section{Consent for publication \\ Not applicable.}

\section{Competing interests}

All the authors, including the members of the Italian Cystic Fibrosis Lung

Transplantation Group, declare that they have no competing interests.

\section{Publisher's Note}

Springer Nature remains neutral with regard to jurisdictional claims in published maps and institutional affiliations.

\footnotetext{
Author details

${ }^{1}$ Infectious Diseases Unit, Azienda Ospedaliero-Universitaria Careggi, Largo Brambilla 3, 50134 Florence, Italy. ${ }^{2}$ Cystic Fibrosis Center, Azienda Ospedaliero-Universitaria di Verona, P.le Stefani 1, 37126 Verona, Italy. ${ }^{3}$ Cystic Fibrosis Center - Adult Unit, Department of Medicine and Infectious Diseases, University Umberto I, Viale Regina Elena 324, 00161 Rome, Italy. ${ }^{4}$ Cystic Fibrosis Center - Adult Unit, Fondazione IRCCS Ca' Granda, Ospedale Maggiore Policlinico, Via F. Sforza 35, 20122 Milan, Italy. ${ }^{5}$ Cystic Fibrosis Center, Meyer Children's Hospital, Viale Pieraccini 24, 50139 Florence, Italy.
} 
Received: 4 January 2018 Accepted: 11 June 2018

Published online: 28 June 2018

\section{References}

1. Hirche TO, Knoop C, Hebestreit H, et al. Practical guidelines: lung transplantation in patients with cystic fibrosis. Pulmonary Medicine 2014; Article ID 621342 (https://doi.org/10.1155/2014/621342).

2. Snell G, Reed A, Stern M, Hadjiliadis D. The evolution of lung transplantation for cystic fibrosis: a 2017 update. J Cyst Fibros. 2017;16:553-64.

3. Liou TG, Adler FR, Cahill BC, et al. Survival effect of lung transplantation among patients with cystic fibrosis. JAMA. 2001;286:2683-9.

4. Mayer-Hamblett N, Rosenfeld M, Emerson J, Goss CH, Aitken ML. Developing cystic fibrosis lung transplant referral criteria using predictors of 2-year mortality. Am J Respir Crit Care Med. 2002;166: 1550-5.

5. Belkin RA, Henig NR, Singer LG, et al. Risk factors for death of patients with cystic fibrosis awaiting lung transplantation. Am J Respir Crit Care Med. 2006;173:659-66

6. Egan M, Edwards LB. Effect of the lung allocation score on lung transplantation in the United States. J Heart Lung Transplant. 2016;35:433-9.

7. Boussaud V, Mal H, Trinquart $L$, et al. One-year experience with highemergency lung transplantation in France. Transplantation. 2012;93:1058-63.

8. Orsini B, Sage E, Olland A, et al. High-emergency waiting list for lung transplantation: early results of a nation-based study. Eur J Cardiothorac Surg. 2014;46:e41-7.

9. Pretagostini R, Peritore D, Rizzato $L$, et al. Urgent lung transplantation national program: the Italian experience. Transplant Proceed. 2013;45:2607-9.

10. Boffini M, Venuta F, Rea F, et al. Urgent lung transplant programme in Italy: analysis of the first 14 months. Interact Cardiovasc Thorac Surg. 2014;19: 795-800.

11. Amato A, Ferrigno L, Salvatore M, Toccaceli V. Italian Cystic Fibrosis Register - Report 2010. Epidemiol Prev. 2016;40(Suppl 2):1-48.

12. Sistema Informativo Trapianti del Centro Nazionale Trapianti: http://www. trapianti.salute.gov.it/cnt/cntStatistiche.jsp?area=cnt-generale\&menu= menuPrincipale\&label $=$ datdoc\&titolo=1111\&etichetta $=$. Accessed 21 Feb 2017.

13. Giordani B, Amato A, Majo F, Ferrari G, Quattrucci S, Minicucci L, Padoan R, Floridia G, Puppo Fornaro G, Taruscio D, Salvatore M. Italian Cystic Fibrosis Registry. Report 2011-2014. Epidemiol Prev. 2018;42(Suppl1):1-32.

14. Thabut G, Christie JD, Mal H, et al. Survival benefit of lung tranplant for cystic fibrosis since lung allocation score implementation. Am J Respir Crit Care Med. 2013;187:1335-40.

15. Vizza CD, Yusen RD, Lynch JP, Fedele F, Patterson A, Trulock EP. Outcome of patients with cystic fibrosis awaiting lung transplantation. Am J Respir Crit Care Med. 2000;162:819-25.

16. Hosendpud JD, Bennett LE, Keck BM, Edwards EB, Novick RJ. Effect of diagnosis on survival benefit of lung transplantation for end-stage lung disease. Lancet. 1998;351:24-7.

17. Gottlieb J, Greer M, Sommerwerck U, et al. Introduction of the lung allocation score in Germany. Am J Transplant. 2014;14:1318-27.

18. Le rapport medical et scientific de l'Agence de la biomedicine, 2013: https://www.agence-biomedecine.fr/annexes/bilan2016/donnes/organes/04coeur-poumon/synthese.htm. Accessed 3 Apr 2017.

19. Braun AT, Dasenbrook EC, Shah AS, Orens JB, Merlo CA. Impact of lung allocation score on survival in cystic fibrosis lung transplant recipients. J Heart Lung Transplant. 2015;34(11):1436-41.

\section{Ready to submit your research? Choose BMC and benefit from:}

- fast, convenient online submission

- thorough peer review by experienced researchers in your field

- rapid publication on acceptance

- support for research data, including large and complex data types

- gold Open Access which fosters wider collaboration and increased citations

- maximum visibility for your research: over $100 \mathrm{M}$ website views per year

At BMC, research is always in progress.

Learn more biomedcentral.com/submissions 